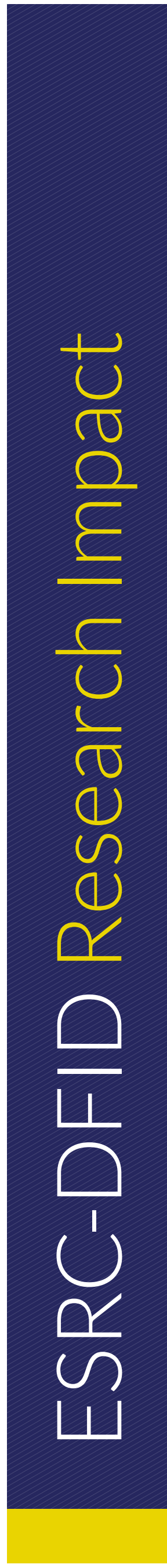

\title{
KEEPING AFRICAN GIRLS IN SCHOOL WITH BETTER SANITARY CARE
}

For young girls in developing countries, not knowing how to manage their periods can hinder access to education. Research from the School of Oriental and African Studies (SOAS), University of London demonstrates that in rural Uganda, providing free sanitary products and lessons about puberty to girls may increase their attendance at school.

\section{THE CHALLENGE}

In many poor communities, menstruation is still often seen as an embarrassing, shameful, and dirty process. Such taboos around the topic mean many adolescent girls are often unprepared for their periods and how to manage them. Less than half of girls in lower- and middle-income countries have access to basics such as sanitary towels or tampons, soap and water, or facilities to change, clean, or dispose of hygiene products.

In Uganda, only 22 per cent of girls are enrolled in secondary schools compared with 91 per cent in primary schools, with those living in rural areas being the least likely group to go to school. Researchers believe that the cost of hygiene products and the difficulties in managing periods play a key role in keeping girls out of school.

\section{THE RESEARCH}

Over 24 months, a trial was conducted in partnership with Plan International Uganda across eight schools, involving 1,008 girls, in Uganda's Kamuli District, an area that had been observed as having low learning levels as well as gender disparity in health and education.

\begin{abstract}
The research tested whether school attendance improved when girls were given (a) reusable sanitary pads, (b) adolescent reproductive health education, (c) neither, or (d) a combination of both. Girls were provided with AFRIpads, a washable, reusable cloth pad produced in Uganda, and locally trained community health nurses held sessions that covered changes which occur during puberty, menstruation, and early pregnancy, and on the prevention of HIV.

Researchers found that better sanitary care and reproductive health education for poor schoolgirls, delivered over two years, did appear to improve attendance. On average, girls increased their attendance by 17 per cent, which equates to 3.4 days out of every 20 days. .

Many girls don't know about periods before they encounter their first one. They are totally unprepared because they receive no information or training on how to manage them. Simple interventions like these can have major long-term economic implications for women in low- and middle-income countries. Catherine Dolan, SOAS, University of London
\end{abstract}

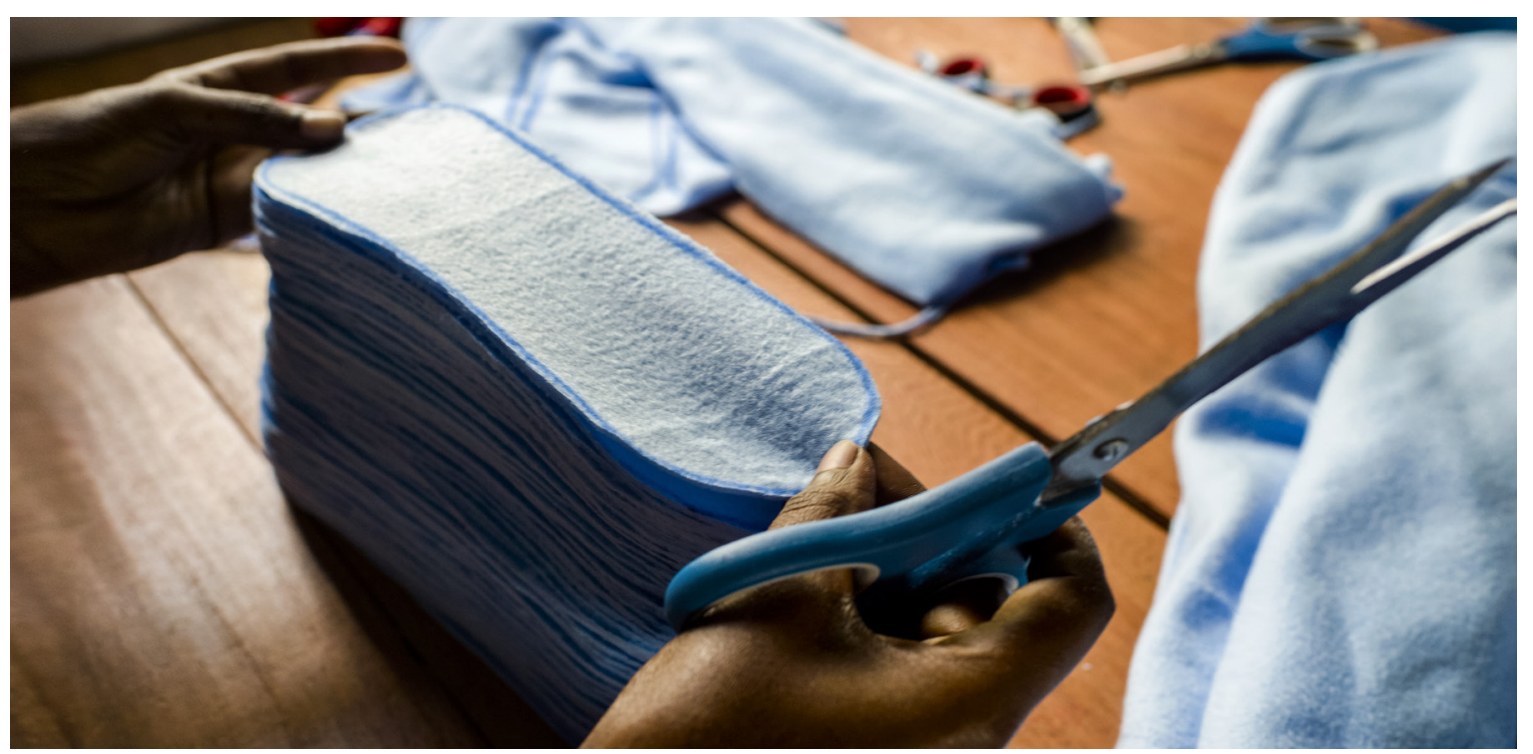

Cover photo: Uganda, Kitengeesa. A worker trims and stacks sanitary pads before they are lined and sewn at the AFRIpads factory. Started by volunteers in 2009, AFRI pads manufactures reusable fibre sanitary pads. Credit: Nyani Quarmyne/Panos. 


\section{THE IMPACT}

The research project has significantly strengthened awareness that sanitary pad provision and puberty education are both vital in improving attendance. Even in the absence of resources to provide sanitary pads, the research recommends that inclusion of adequate and gender-sensitive puberty education in the school curriculum can improve attendance.

\section{Organisations such as UNICEF and the NGO CARE} have used the evidence to identify solutions to barriers to girls' schooling associated with puberty.

\section{.}

These findings will make an important contribution to CARE's efforts to fight poverty by removing the barriers that keep girls out of school. All over civil society, in fact, bigger funding will now be available for large-scale rollouts because of the hard evidence provided by the study in Uganda.

Joan Garvey Lundgren, Executive Director, Strategic Partnerships, CARE USA

The project collaborated with Save the Children, the United Nations Educational, Scientific and Cultural Organization (UNESCO), WaterAid, and AFRIpads to lobby for menstrual hygiene management to be included as an indicator in post-2015 sustainability goals. Further collaborations building on the evidence have included working with Save the Children on how to link the distribution of sanitary care to their West African programmes, and with UNESCO on effective programming in puberty education and menstrual hygiene management.

Ghana's Deputy Minister of Education referenced the research when defending the decision to allocate part of the country's 2014 World Bank loan to providing sanitary pads for female students in need. Samuel Okudzeto Ablakwa stated that when adolescent girls are unable to take proper care of themselves during the menstruation period, it affects their confidence, which eventually keeps them out of school.

The research team continues to use the results as part of a push to promote female hygiene onto the global development agenda. The findings featured in preparatory documents for the World Health Organization (WHO)/ UNICEF Joint Monitoring Programme indicators for menstrual hygiene management, and have been cited in the UNESCO Puberty Education \& Menstrual Hygiene Management report, which aims to promote sexuality education as part of skills-based health education for young people.

The impact of the research has the potential for addressing psychosocial wellbeing, dignity, comfort, and ability to manage menstruation without shame, which are all essential for girls responding to the challenges presented by menstruation in low-income contexts.
Studies such as this are too few and far between. They are critical to give context to the impact of hygiene and sanitation during puberty, which in turn helps us work towards solutions to improve girls' life chances.

Brooke Yamakoshi, Water, Sanitation and Hygiene (WASH) Specialist (Sanitation and Hygiene), United Nations Children's Fund (UNICEF)

\section{FURTHER READING}

Dolan, C.S.; Ryus, C.R.; Dopson, S.; Montgomery, P. and Scott, L. (2014) 'A Blind Spot in Girls' Education: Menarche and its Webs of Exclusion in Ghana', Journal of International Development 26.5: 643-57

Hennegan, J.; Dolan, C.; Steinfield, L. and Montgomery, P. (2017) 'A Qualitative Understanding of the Effects of Reusable Sanitary Pads and Puberty Education: Implications for Future Research and Practice', Reproductive Health 14.78

Hennegan, J.; Dolan, C.; Wu, M.; Scott, L. and Montgomery, P. (2016) 'Measuring the Prevalence and Impact of Poor Menstrual Hygiene Management: A Quantitative Survey of Schoolgirls in Rural Uganda', BMJ Open 6.12: 1-14

Montgomery, P.; Ryus, C.R.; Dolan, C.S.; Dopson, S. and Scott, L.M. (2012) 'Sanitary Pad Interventions for Girls' Education in Ghana: A Pilot Study', PLoS ONE 7.10: e48274

Montgomery, P.; Hennegan, J.; Dolan, C.; Wu, M.; Steinfield, L. and Scott, L. (2016) 'Menstruation and the Cycle of Poverty: A Cluster Quasi-Randomised Control Trial of Sanitary Pad and Puberty Education Provision in Uganda', PLoS ONE 11.12: e0166122

Hennegan, J.; Dolan, C.; Wu, M.; Scott, L. and Montgomery, P. (2016) 'Schoolgirls' Experience and Appraisal of Menstrual Absorbents in Rural Uganda: A Cross-Sectional Evaluation of Reusable Sanitary Pads', Reproductive Health 13.1: 143

Hennegan, J. and Montgomery, P. (2016) 'Do Menstrual Hygiene Management Interventions Improve Education and Psychosocial Outcomes for Women and Girls in Low and Middle Income Countries? A Systematic Review', PLoS ONE 11.2: e0146985

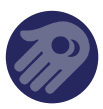

Menstruation and the Cycle of Poverty: Does the provision of sanitary pads improve the attendance and educational outcomes of girls in school?

The research team was funded by ESRC-DFID's Joint Fund for Poverty Alleviation Research, led by Catherine Dolan, SOAS, University of London; Paul Montgomery, University of Birmingham; and Linda Scott, Chatham House. The research was carried out in partnership with Plan International Uganda, with the assistance of Julie Hennegan, Johns Hopkins University; Maryalice Wu, University of Illinois; and Laurel Steinfield, Bentley University.

\section{THE IMPACT INITIATIVE}

\section{For International Development Research}

The Impact Initiative seeks to connect policymakers and practitioners with the world-class social science research supported by the ESRC-DFID Strategic Partnership, maximising the uptake and impact of research from: (i) the Joint Fund for Poverty Alleviation Research, and (ii) the Raising Learning Outcomes in Education Systems Programme. We seek to identify synergies between these programmes and their grant holders, support them to exploit influencing and engagement opportunities, and facilitate mutual learning. The Impact Initiative is a collaboration between the Institute of Development Studies (IDS) and the University of Cambridge's Research for Equitable Access and Learning (REAL) Centre.

All content is available under the Open Government License v3.0, except where otherwise stated.

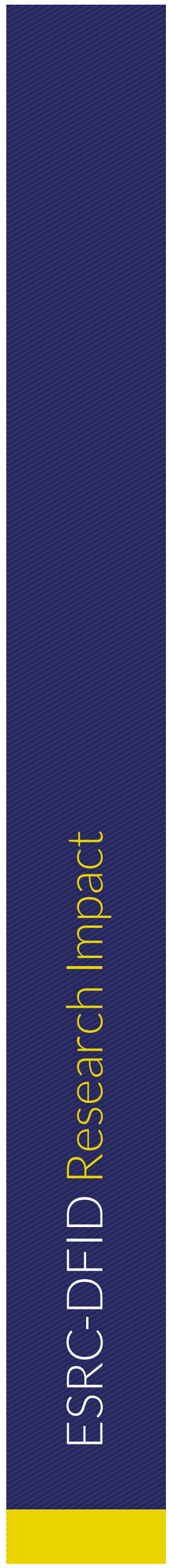

